\title{
DESIGN AND MODELING OF A TRIPLE-STEPPED BEAM WITH OUT-OF-PLANE MOTION FOR BISTABLE MICROSWITCH APPLICATIONS
}

\author{
Duong Ngoc Bich ${ }^{1}$, Truong Van Men², Duong Minh Hung ${ }^{3}$
}

\begin{abstract}
Microswitches have been used for many different applications in building, automation, and security due to requiring little force. A novel design of a triple-stepped beam structure for a mechanical bistable microswitch is presented, and it was found that the bistability of the beam can be achieved by applying an electrostatic force which allows a high deflection with small electrode separation. A finite element method analysis has been used to design the bistable microswitch in a certain range of geometries based on the standard of Taiwan Semiconductor Manufacturing Company (TSMC). The simulation results show that the device requires a very low input force to get to the bistable stages. The maximum force and the minimum force for switching between the bistable stages are $0.85 \mathrm{mN}$ and $0.23 \mathrm{mN}$, respectively, which is suitable for electrostatic force at a microscale. The bistability is obtained with the second equilibrium at $75.17 \mu m$ that guarantees the perfect contact location between the beam and the conduction path $(N+)$ located at $65.45 \mu \mathrm{m}$.
\end{abstract}

Keywords: triple-stepped beam structure, bistable micromechanism, bistable microswitch, electrostatics microswitch.

\section{INTRODUCTION}

Microelectromechanical systems (MEMS) have recently been developed as alternatives for conventional electromechanical devices

\footnotetext{
${ }^{1,2,3}$ Tra Vinh University

Email: ngocbich1184@tvu.edu.vn

Received date: $27^{\text {th }}$ February 2020; Revised date: $22^{\text {nd }}$ July 2020; Accepted date: $14^{\text {th }}$ October 2020
}

such as switches, actuators, valves, and sensors. The use of electrostatic actuation for MEMS is attractive because of the high energy densities and large forces available in microscale devices [1]-[3]. In many designs, the positions of electrodes are controlled by a balance between an electrostatic attractive force and a mechanical restoring force. Bistable micro-mechanisms are gaining more attention in MEMS applications due to their advantages. In general, bistable mechanisms are monolithic devices with two stable equilibrium positions separated by an unstable equilibrium position as illustrated in Figure 1. They have the ability to stay in their positions without an input of energy, and a certain amount of work is required to switch between their positions [4], [5]. One of the outstanding advantages of bistable micro-mechanisms is that no power is required to keep the mechanism in either of its bistable positions and thereby, reduces energy consumption [6]. A bistable mechanism can meet requirements of low actuation force and power, high cycle life, and predictable, repeatable motion in MEMS applications [7] which is why bistable mechanisms have been intensively studied for microswitch applications. However, in microscale, the fabrication method and how to make the bistable mechanism jump between its stable positions effectively are the challenges of designing bistable microswitches.

\section{LITERATURE REVIEW}

Recently, various approaches for micromechanical bistable switches have been studied. For instance, Lisec et al. was one of the 


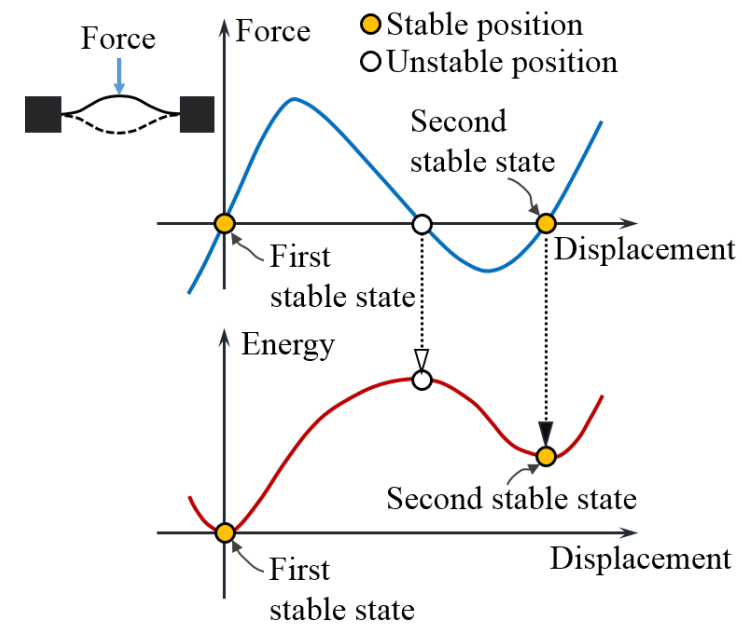

Fig. 1: Force and energy versus displacement curves of a typical bistable mechanism [8]

first to present a bistable pneumatic microswitch for driving passive fluidic components. The tested device exhibited high efficiency and low gas consumption [9]. Vangbo et al. [10] fabricated a lateral symmetrically bistable buckled beam for snap-in holding structures by deep silicon reactive ion etching using the black silicon method, subsequently released and thermally oxidized. Matthew et al. [11] reported a micro-bistable mechanism used in microswitches and microvalves, where the bistability of the device with a fully-compliant mechanism was designed and optimized based on the pseudorigid-body model. Its operation was frictionfree, with no backlash or wear due to no rigid-body joints. Qiu et al. [12] fabricated a micro-bistable mechanism using DRIE (deep reactive ion etching) that has a curved shape but no residual stress. It is observed that the tested behavior of the micro-scale mechanism followed the theoretical and numerical predictions by using a compressed buckled beam [13]. Which proved that the snap-through mechanism and the maximum force can be analytically predicted. In general, bistability is achieved in all these cases by specialshaped beams in combination with a snap- ping mechanism.

An alternative has been developed where an electrostatically driven bistable switch has been based on a mechanically pre-stressed toggle-lever. Inbar et al. [14] proposed a mechanism that converts in-plane motion into out-of-plane motion, which is fully compatible with standard mass fabrication methods. The mechanism applies the well-established in-plane actuation achieved by comb-drives and converts it into an out-of-plane motion. Inbar et al. [14] also presented new devices that were specifically designed to demonstrate the tunability of the conversion ratio. Furthermore, on a bistable switch based upon electrostatic force, Rob et al. [15] presented an electrostatic actuator design where a deformable mechanical structure is bent around a fixed curved electrode by means of electrostatic forces. Building upon this Hung et al. [16] examined the leveraged bending and strain-stiffening methods for extending the stable travel range of electrostatic actuators beyond the $1 / 3$ of the gap pull-in instability limit for elastically suspended parallelplate electrostatic actuators. This work also demonstrated how strain-stiffened actuator designs can be optimal for achieving a given travel distance while minimizing actuation voltage. Lior et al. [17] developed a twodirectional bistable microswitch actuated by a single electrode. The snap-through switching of the device was actuated by preloading the structure using a rising voltage applied to the electrode, followed by a sudden decrease of the voltage. Additionally Miao et al. presented a large out-of-plane bistable microswitch actuated by an electromagnetic force. The bistability was obtained by balancing the magnetic force and elastic force.

From the literature, it is obvious that a number of attempts have been made to design bistable microswitches using different actuation and fabrication processes in order to meet different applications. In addition, modeling is considered as an effective approach to predict structure behaviors un- 
der working conditions due to the distinct fabrication in microscale. In this work, we proposed a novel design of a triple stepped beam structure for a bistable microswitch. The main constraints of the design are how to adopt the standard of Taiwan Semiconductor Manufacturing Company (TSMC) and low required maximum force that can be actuated by an electrostatic force in microscale. The finite element method (using commercial ABAQUS software) is employed to analyze the force-displacement and stressdisplacement relations when the structure is loaded by an input displacement to obtain an out-of-plane motion, actuation force and the bistability of the triple stepped beam as well as its dimensions in the range of the TSMC standard.

\section{STRUCTURAL DESIGN AND SIMULATION}

Our design of an out-of-plane actuation structure is based on a triple stepped beam as depicted in Figure 1 presenting the operating principle of the device. Firstly, when the voltage input is initially applied through the out-of-plane beam at one fixed end with the cathode side and bottom electrode with the anode side as illustrated in Figure 1 (a). The stepped beam is then moved down towards to the conduction path by the presence of the electrostatic force $(F)$. The beam reaches the bistability at the contact position and becomes the conductive line. Because of the bistability, the device is always at contact location even when voltage input is removed (as depicted in Figure 1 (b). Finally, the voltage input is again applied through the fixed end of the stepped beam with the anode side and the top anode electrode to move the beam upward as well as return it to the initial stage termed in the open stage as shown in Figure 1 (c).

The novel out-of-plane actuation structure is operated by electrostatic force with the full of dimension is illustrated in Figure 2.
The height of the structure and the width of each layer stepped beam are fixed based on the TSMC 2P4M standard (Taiwan Semiconductor Manufacturing Company 2 polysilicon layers 4 metal layers) with a die-cast housing area of $500.500 \mu \mathrm{m}^{2}$ in the design of the microswitch. Based on this standard, the distance between the top electrode and the bottom electrode is $65.45 \mu \mathrm{m}$ and the total length of the stepped beam is $335 \mu \mathrm{m}$. The beam thickness is $3.0 \mu \mathrm{m}$. The first step of this work was to design and simulate the bistable beam in order to obtain the desired force and displacement. The finite element method (ABAQUS) was utilized for this purpose. A three-dimensional model (3D) with a CPE4R element type is employed in the force-displacement and stress-displacement analyses. During the simulation, both ends of the beam are set as anchors. The input displacement is imposed on the middle point of the beam in the y-direction. The force and stress versus the displacement are obtained after the simulation is completed. The triple stepped beam consists of two kinds of materials, aluminum and tungsten. In particular, all the horizontal segments are made of aluminum while the vertical segments are tungsten. These material properties used for the simulation are given in Table 1. Undeformed and deformed finite element meshes for the stepped beam structure are depicted in Figure 3. A close-up view of the mesh near the fixed end of the beam is also shown in the figure. A study of the mesh convergence was initially carried out to obtain accurate solutions. Based on this, the meshing element size of $0.5 \times 0.5 \times 0.5 \mu \mathrm{m}$ is used for all following analyses.

\section{SIMULATION RESULTS AND DISCUSSION}

The main focus of this work is on how to achieve the force-displacement relation of the triple stepped beam which is applicable for an electrostatic microswitch in the constraints 

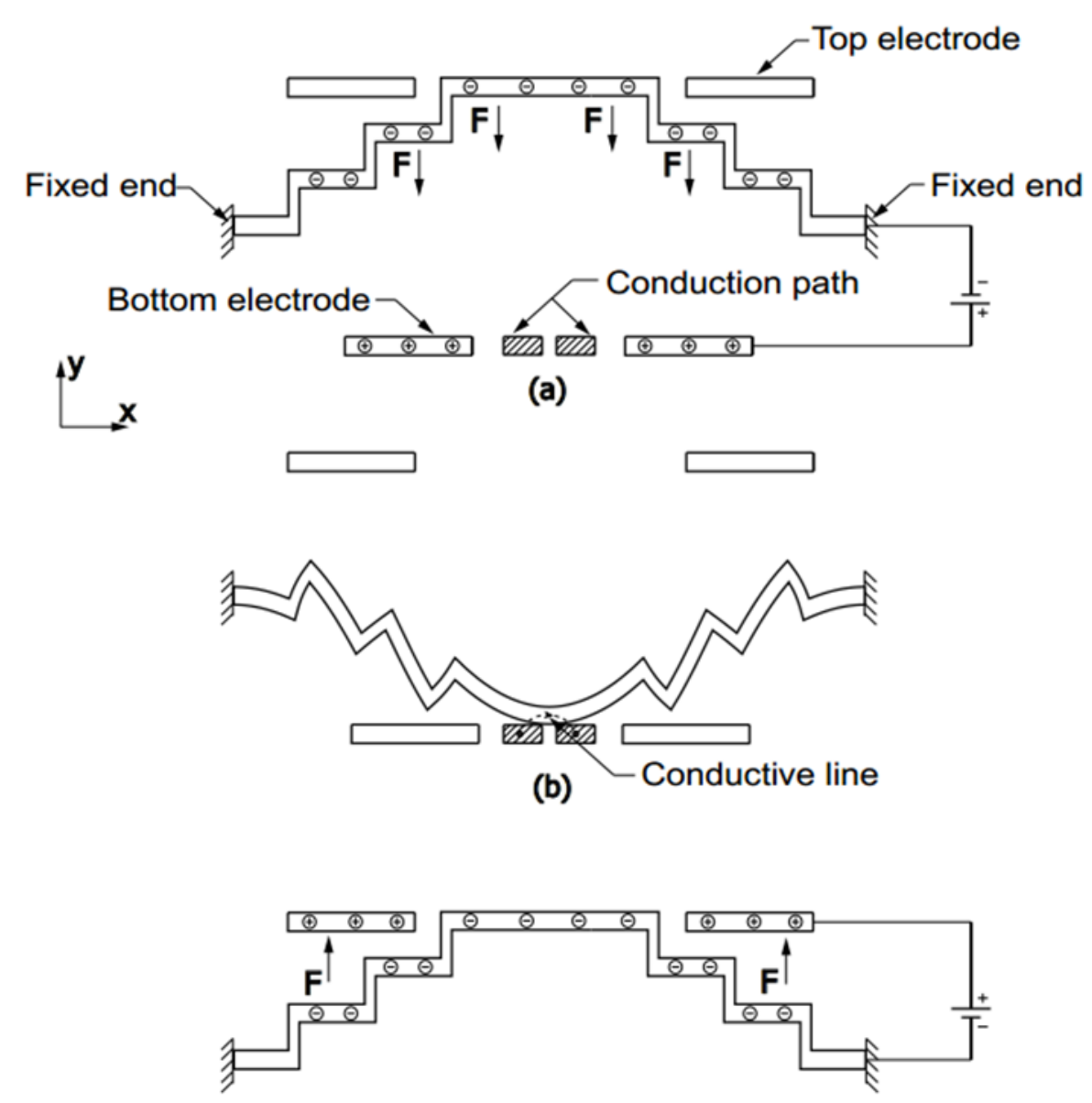

पाय

(c)

Fig. 2: Schematic of bistable switch at initial position (a), closed position (b) and open position (c)

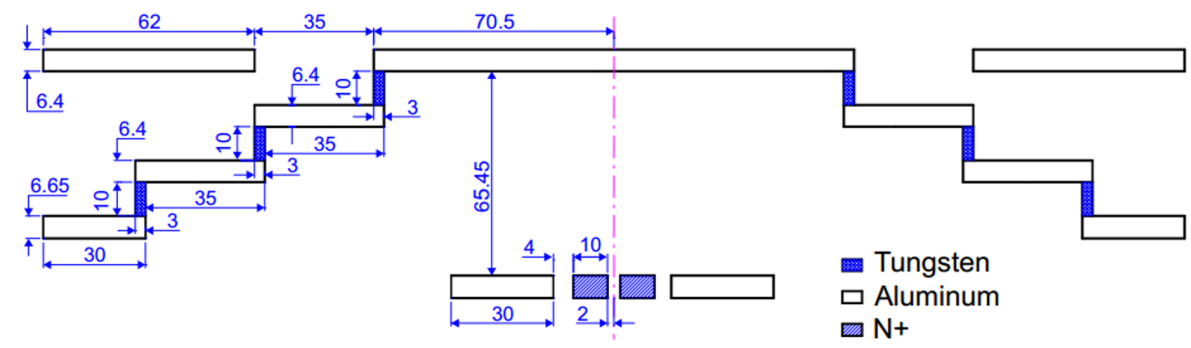

Fig. 3: Dimensions of the triple stepped beam 
Table 1: The material properties of triple stepped beam

\begin{tabular}{llll}
\hline Material & $\begin{array}{c}\text { Young's Modulus } \\
(\mathrm{MPa})\end{array}$ & $\begin{array}{c}\text { Poision Ratio } \\
(-)\end{array}$ & $\begin{array}{c}\text { Density } \\
(\mathrm{g} / \mu \mathrm{m} 3)\end{array}$ \\
\hline Aluminum & $80 \times 10^{3}$ & 0.35 & $2.7 \times 10^{-12}$ \\
\hline Tungsten & $70 \times 10^{3}$ & 0.28 & $19.25 \times 10^{-12}$ \\
\hline
\end{tabular}

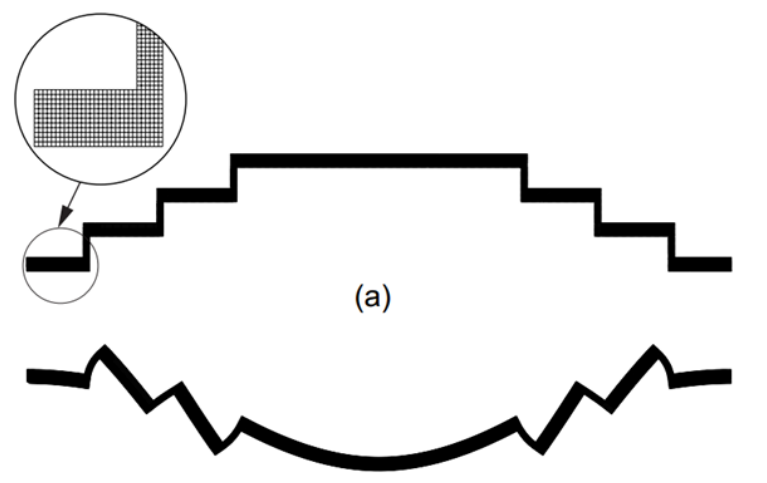

(b)

Fig. 4: A mesh of triple stepped beam: (a) The beam at the initial stage without the deformation and (b) the beam at the open stage with the deformation

of the TSMC 2P4M. For a feasible solution, the design process is based on the trial and error method which means that the size of each segment including its length, height, and width is changed in order to obtained the bistable mechanism with the feasible displacement, input force, and induced stress. The final model of the triple stepped beam is presented in Figure 3. The deformation contour plot of the triple stepped beam is shown in Figure 5 as the middle point of the beam is displaced downward by $75.17 \mu \mathrm{m}$ obtained by finite element analysis. The vertical segments undergo more deformation than the horizontal segments due to the smaller width. The results in the maximum stress induced in these beams as observed in Figure 8. The force-displacement curve of the triple stepped beam is shown in Figure 6, and it is noticeable from this figure that the triple stepped beam behaves as a bistable mechanism and the second stable position occurs at a distance of $75.17 \mu \mathrm{m}$. The value of maximum force $(854 \mu \mathrm{N})$ is about 3.5 times larger than that of the minimum force $(-232.5$ $\mu \mathrm{N})$. During operation, a force greater than the maximum force should be applied for enough time to pass over the neutral position at $60 \mu \mathrm{m}$ (unstable position) in order to reach the second stable position. In contrast, a force larger than minimum force value is needed to make the bistable mechanism return from the second stable position to the first stable position. The magnitudes of these forces are small enough to be driven by the electrostatic effect [18]. Furthermore, since the conductive path $(\mathrm{N}+)$ located at a distance of $65.45 \mu \mathrm{m}$ as regarded in Figure 3 and the distance is shorter than that of the second equilibrium position (as seen in Figure 6), the bistable microswitch will provide good contact between the stepped beam and the conduction path. It is also noted that the distance between the middle horizontal segment and the conduction path should be designed to be larger than the displacement of the middle horizontal segment, where the minimum force occurs, in order to guarantee that the beam will always move toward the second stable position. The

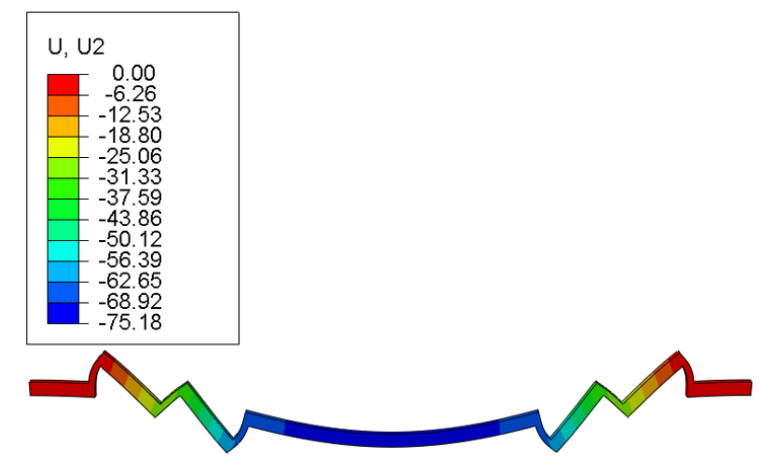

Fig. 5: The contour plot of the displacement $(\mu \mathrm{m})$ of the triple stepped beam at the position of $75.17 \mu \mathrm{m}$

accurate determination of the internal stress is important for structural design purposes, 


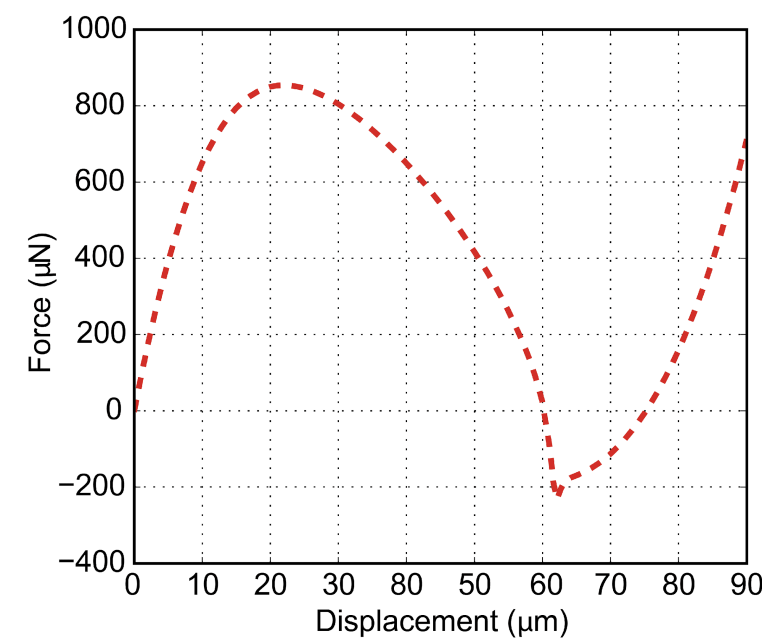

Fig. 6: Force-displacement curve of the triple stepped beam

particularly, for miniaturization and for identifying the design limits under elastic or failure limits. The stress-displacement relation that corresponds to the force-displacement curve is shown in Figure 7. When the beam moved downward to a position at about 62 $\mu \mathrm{m}$, the maximum stress is induced up to 6.85 $\mathrm{MPa}$ at some maximum bent locations of the beam which is much smaller the yield strengths of tungsten (550 $\mathrm{MPa})$ and aluminum (140 MPa), confirming that the designed beam is strong enough under the simulated working conditions. The amount of stress in the beam gradually reduces after the beam passes through the depth of 62 $\mu \mathrm{m}$ as seen in this figure. The concentrated stresses are only occurred at the corners of the vertical beams at the contact point $(65.45$ $\mu \mathrm{m})$ as observed in Figure 8.

\section{CONCLUSION}

A novel type of a bistable microswitch using a triple stepped beam has been proposed and analyzed. Based on the simulation results, the maximum force is small at around $0.85 \mathrm{mN}$. The bistability is obtained with a second equilibrium at $75.17 \mu \mathrm{m}$ which guaranties the perfect contact location between the beam and the conduction path $(\mathrm{N}+)$

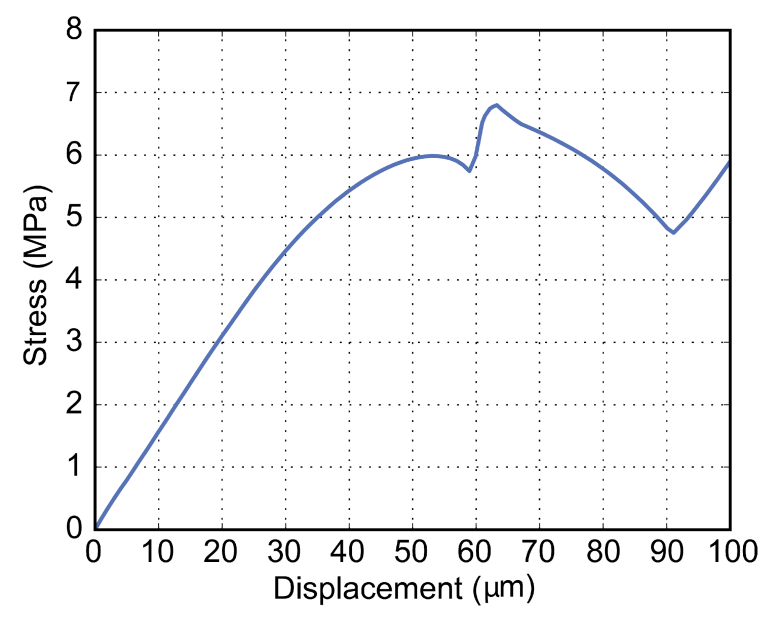

Fig. 7: Stress-displacement curve of the triple stepped beam

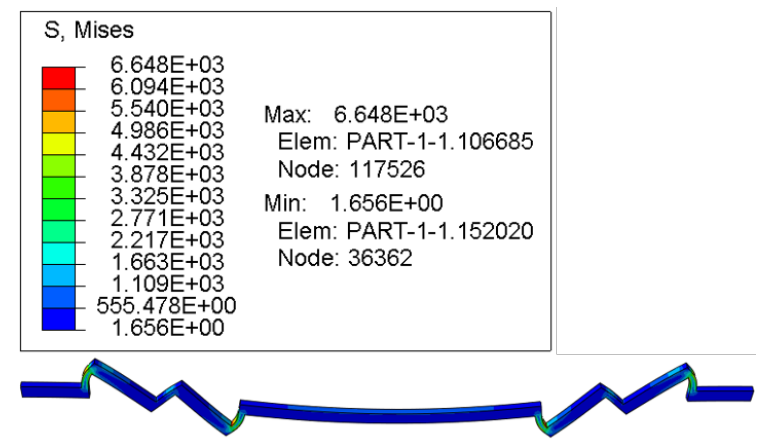

Fig. 8: The contour plot of Mises stress (kPa) in the triple stepped beam at the position of $65.45 \mu \mathrm{m}$

located at $65.45 \mu \mathrm{m}$. The concept allows for the compensation of reaction forces of the load during switching by the appropriate design, thus reducing the need for electrostatic switching forces. In addition, the stress analysis demonstrates that Mises stress is much lower than the yield strengths of the selected materials. The design structure can be fabricated by the TSMC $2 \mathrm{P} 4 \mathrm{M}$ process. In future work, we will demonstrate the outof-plane motion of the bistable mechanism by applying the electrostatic force and the compatibility of applied voltage and the electrostatic force. 


\section{REFERENCES}

[1] Karumuri SR, Sravani G, Thalluri LN. A micro level electrostatically actuated cantilever and metal contact based series RF MEMS switch for multi-band applications. Cogent Engineering. 2017;4.

[2] Chu C-H, Wen-Pin S, Chung S-Y, Tsai H-C, Shing T, Chang P-Z. A low actuation voltage electrostatic actuator for RF MEMS switch applications. J Micromech Microeng. 2007;17:1649-56.

[3] Kim M-W, Song Y-H, Ko S-D, Ahn S-J, Yoon JB. Ultra-low voltage MEMS switch using a folded hinge structure. Micro and Nano Systems Letters. 2014;2(1):2.

[4] Milojevic A. Compliant bistable mechanisms; 2011:181-186.

[5] Jin Q, Lang JH, Slocum AH. A curved-beam bistable mechanism. Journal of Microelectromechanical Systems. 2004;13(2):137-46.

[6] Casals-Terré J, Fargas-Marques A, Shkel AM. SnapAction Bistable Micromechanisms Actuated by Nonlinear Resonance. Journal of Microelectromechanical Systems. 2008;17:1082-93.

[7] Wilcox DL, Howell LL. Fully compliant tensural bistable micromechanisms (FTBM). Journal of Microelectromechanical Systems. 2005;14(6):1223-35.

[8] Han JS, Müller C, Wallrabe U, Korvink JG. Design, Simulation, and Fabrication of a Quadstable Monolithic Mechanism With X- and Y-Directional Bistable Curved Beams. Journal of Mechanical Design. 2006;129(11):1198-203.

[9] Lisec T, Kreutzer M, Wagner B. A bistable pneumatic microswitch for driving fluidic components. Sensors and Actuators A: Physical. 1996;54(1):746-9.
[10] Vangbo M, Bäcklund Y. A lateral symmetrically bistable buckled beam. Journal of Micromechanics and Microengineering. 1998;8(1):29-32.

[11] Jensen BD, Parkinson MB, Kurabayashi K, Howell L, Baker MS. Design optimization of a fully-compliant bistable micro-mechanism. ASME International Mechanical Engineering Congress and Exposition, Proceedings. 2001;2:2931-7.

[12] Qiu J, Lang JH, Slocum A. A centrally-clamped parallel-beam bistable MEMS mechanism; 2001.

[13] Vangbo M. An analytical analysis of a compressed bistable buckled beam. Sensors and Actuators A: Physical. 1998;69(3):212-6.

[14] Hotzen I, Ternyak O, Shmulevich S, Elata D. Massfabrication compatible mechanism for converting inplane to out-of-plane motion. Proceedings of the IEEE International Conference on Micro Electro Mechanical Systems (MEMS). 2015;2015:897-900.

[15] Legtenberg R, Gilbert J, Senturia S, Elwenspoek M. Electrostatic Curved Electrode Actuators. Journal of Microelectromechanical Systems. 1997;6(3):257-65.

[16] Hung ES, Senturia SD. Extending the travel range of analog-tuned electrostatic actuators. Journal of Microelectromechanical Systems. 1999;8(4):497-505.

[17] Medina L, Gilat R, Ilic R, Krylov S. Two-Directional Operation of Bistable Latchable Micro Switch Actuated by a Single Electrode; 2017.

[18] Chuang W-C, Lee H-L, Chang P-Z, Hu Y-C. Review on the modeling of electrostatic MEMS. Sensors (Basel, Switzerland). 2010;10(6):6149-71. 